\title{
Retrasplante cardiaco electivo. Primer caso en México
}

\author{
Guillermo Careaga-Reyna y Hugo Jesús Zetina-Tun \\ Instituto Mexicano del Seguro Social, Centro Médico Nacional La Raza, Hospital General, Ciudad de México, México
}

\begin{abstract}
Resumen
El retrasplante cardiaco ortotópico se utiliza para tratar la falla cardiaca terminal del injerto cardiaco trasplantado. Presentamos el primer caso exitoso de retrasplante cardiaco electivo en México. Se trató de un varón de 25 años con trasplante de corazón, quien presentó vasculopatía crónica resistente del injerto. Fue retrasplantado electivamente en septiembre de 2017; las complicaciones durante la evolución posoperatoria fueron tratadas con respuesta favorable. Egresó por mejoría a las cuatro semanas del posoperatorio. Se concluye que en los casos apropiadamente seleccionados y valorados integralmente, el retrasplante cardiaco es una opción adecuada para el manejo de la falla cardiaca del injerto.
\end{abstract}

PALABRAS CLAVE: Retrasplante cardiaco. Rechazo crónico. Inmunosupresión. Falla cardiaca crónica. Vasculopatía del injerto cardiaco.

\begin{abstract}
Orthotopic cardiac retransplantation is used to treat transplanted cardiac graft end-stage failure. We present the first case of successful elective cardiac retransplantation in Mexico. It was a 25-year old male with heart transplantation who developed graft-resistant chronic vasculopathy. He underwent elective retransplantation in September 2017; complications during postoperative evolution were treated with favorable response. He was discharged owing to improvement at four weeks postoperatively. It is concluded that in adequately selected cases and comprehensively assessed, cardiac retransplantation is an appropriate option to treat cardiac graft failure.
\end{abstract}

KEY WORDS: Cardiac retransplantation. Chronic rejection. Immunosuppression. Chronic heart failure. Cardiac allograft vasculopathy.

\section{Introducción}

El trasplante de corazón ha demostrado a lo largo de décadas que es la opción ideal para el tratamiento definitivo de la falla cardiaca terminal de diversas causas; con la terapia inmunosupresora actual, la supervivencia y la calidad de vida de los pacientes se ha incrementado, ${ }^{1}$ aunque a largo plazo la mortalidad continua por complicaciones crónicas como la vasculopatía del injerto, sobre todo a cinco años postrasplante. ${ }^{2}$

La vasculopatía del injerto es una enfermedad caracterizada por la aparición de lesiones coronarias múltiples obstructivas, difusas, excéntricas, la mayoría de las veces no manejables mediante cardiología intervencionista. ${ }^{3}$ Una vez diagnosticada se utilizan diversas formas de tratamiento como inmunosupresión agresiva, intervencionismo coronario percutáneo con colocación de stents e, incluso, cirugía de revascularización coronaria. Sin embargo, estas modalidades pueden no ser suficientes y las lesiones progresan y se incrementa la falla cardiaca, dejando como única opción al retrasplante cardiaco. ${ }^{4}$

El retrasplante cardiaco representa hasta $3 \%$ de todos los trasplantes cardiacos en el mundo ${ }^{4}$ y se indica para tratar la vasculopatía crónica del injerto (37\%), disfunción temprana del injerto (32\%) y rechazo agudo del
Correspondencia:

Guillermo Careaga-Reyna

E-mail: gcareaga3@gmail.com
Fecha de recepción: 19-12-2017

Fecha de aceptación: 23-04-2018

DOI: 10.24875/GMM.18004054
Gac Med Mex. 2018;154:617-619

Disponible en PubMed www.gacetamedicademexico.com 
injerto cardiaco (14\%). ${ }^{5,6}$ La supervivencia es $4 \%$ menor que en el primer trasplante a un año y $70 \%$ versus $80 \%$ a tres años. ${ }^{4}$ La supervivencia es menor si el retrasplante se realiza los primeros dos años después del primer trasplante ${ }^{6,7}$ principalmente cuando el intervalo es menor de seis meses entre los dos trasplantes. Los factores de riesgo en el retrasplante son la edad $>60$ años en los receptores y donadores de sexo femenino. Las complicaciones perioperatorias y posoperatorias de mediano y largo plazo son similares que en el primer trasplante y la supervivencia de los pacientes sometidos a trasplante cardiaco por primera vez y a retrasplante es similar. ${ }^{8,9}$

Presentamos la experiencia con el primer retrasplante electivo de corazón realizado en México.

\section{Caso clínico}

Hombre de 25 años, quien el 20 de octubre de 2009 recibió trasplante de corazón ortotópico bicaval como urgencia nacional por presentar falla cardiaca terminal resistente e irreversible, secundaria a cardiomiopatía dilatada idiopática de un año de evolución, con recuperación y egreso hospitalario al décimo día del posoperatorio. Su manejo inmunosupresor fue con esteroides, micofenolato mofetilo y tacrolimus, con evolución clínica satisfactoria, en clase funcional I de la escala NYHA (New York Heart Association) hasta enero de 2016, cuando inició con deterioro hasta llegar a clase funcional III NYHA y bradicardia sinusal.

Por histopatología se documentó rechazo agudo $1 \mathrm{R}$ de la clasificación de la International Society for Heart and Lung Transplantation (ISHLT), motivo por el cual se ajustó el tratamiento inmunosupresor a rangos terapéuticos. En mayo de 2016 tuvo síncope y el electrocardiograma mostró bloqueo auriculoventricular de segundo grado, con pausas sinusales, además de una fracción de expulsión del ventrículo izquierdo (FEVI) de $40 \%$ y dilatación del ventrículo izquierdo a $54 \mathrm{~mm}$. Se decidió implante de marcapaso unicameral, con el que se observó mejoría de la clase funcional a II NYHA. En enero de 2017 presentó periodos de taquicardia supraventricular, tratada con amiodarona oral; tres meses después se observó deterioro de clase funcional a III NYHA, edema de piernas, disnea y ortopnea ocasional, taquiarritmias, pérdida de peso de $5 \mathrm{~kg}$ en seis semanas. Con un nuevo ecocardiograma se registró FEVI de 40 \%, diámetro diastólico del ventrículo izquierdo de $56 \mathrm{~mm}$, desplazamiento del anillo tricuspídeo (TAPSE) de $12 \mathrm{~mm}$, con tronco de la arteria pulmonar (TAP) 34/18 y presión sistólica arterial pulmonar (PSAP) de 19 mm Hg. El Holter indicó episodios de taquicardia supraventricular y taquicardia ventricular autolimitadas. Se realizó coronariografía y toma de biopsia endomiocárdica, que mostraron enfermedad plurivascular con lesiones difusas y rechazo agudo con clasificación $2 \mathrm{R}$ de la ISLHT. Con estos datos se confirmó la vasculopatía del injerto de grado severo.

Se colocó un stent medicado a la arteria coronaria descendente anterior y en mayo del mismo año se sustituyó el marcapaso unicamaral por un desfibrilador automático implantable. Se planteó la necesidad de efectuar retrasplante de corazón y en preparación al mismo, se midieron niveles de panel reactivo de anticuerpos (PRA): I de $22 \%$ y II de $77 \%$. El paciente fue sometido a desensibilización de anticuerpos con $1 \mathrm{~g} / \mathrm{kg} / \mathrm{semana}$ de inmunoglobulina $\mathrm{G}$ intravenosa, por cuatro dosis, seguida de rituximab, $375 \mu \mathrm{g} / \mathrm{m}^{2}$ de superficie corporal.

Concluida la desensibilización y ante la presencia de donador compatible, se efectuó retrasplante de corazón el 18 de agosto del 2017. El abordaje quirúrgico se llevó a cabo en la esternotomía previa, la cual se encontró tipo III, con lisis de adherencias, colocación de sistema de derivación cardiopulmonar, retiro de injerto cardiaco e implante del nuevo corazón, con un tiempo de isquemia total del injerto de 305 minutos; la derivación cardiopulmonar fue de 265 minutos y el pinzamiento aórtico de 81 minutos. En el posoperatorio, el paciente cursó con hemorragia mayor de la habitual, falla renal aguda AKIN III, que requirió terapia renal sustitutiva con diálisis peritoneal por 72 horas. El ecocardiograma mostró disfunción biventricular predominantemente derecha, tratada con inotrópicos y vasopresores. La ventilación mecánica se retiró al noveno día de la cirugía. Por datos de neumonía nosocomial y crecimiento en cultivo de Candida albicans recibió caspofungina por 14 días. El paciente permaneció en terapia intensiva posquirúrgica durante 13 días y egresó del hospital a los 22 días del procedimiento. El ecocardiograma de egreso mostró FEVI de $65 \%$, TAPSE de $15 \mathrm{~mm} \mathrm{Hg}$ y mínimo derrame pericárdico. Para inmunosupresión se utilizaron $2.4 \mathrm{mg} /$ $\mathrm{kg}$ de timoglobulina distribuidos en tres tomas, que se complementaron con esteroides, micofenolato de mofetilo y sirolimus. El último nivel de sirolimus fue de $4 \mathrm{nmol} / \mathrm{L}$. La biopsia endomiocárdica a los 45 días no mostró datos de rechazo y al momento de este informe el paciente se encontraba en clase funcional I NYHA.

\section{Discusión}

La mejoría en los resultados del trasplante de corazón lo convierten en la mejor opción terapéutica en la 
insuficiencia cardiaca terminal; lo anterior se debe a la selección de los pacientes, la inmunosupresión, la experiencia técnica de los grupos médico-quirúrgicos, la preservación de los órganos y el cuidado posoperatorio. ${ }^{1}$

A la mejoría en la supervivencia y calidad de vida se suma la posibilidad de complicaciones relacionadas con la inmunosupresión, como infecciones y rechazo del órgano. Una de las expresiones de este último es la vasculopatía del injerto, que se presenta en $43 \%$ de los estudios angiográficos y hasta en $80 \%$ de los ultrasonidos vasculares, principalmente en niños. ${ }^{10}$ La evolución de esta enfermedad es progresiva y aun con el empleo de otras opciones, como la intervención coronaria percutánea o la cirugía de revascularización, el retrasplante de corazón es la única opción en casos bien seleccionados. ${ }^{5,11-13}$ El primer retrasplante de corazón lo llevó a cabo el grupo de Copeland ${ }^{14}$ en 1977 y desde entonces se ha incrementado la realización de este procedimiento.

El retrasplante de corazón ocupa entre 3 y $5.4 \%$ del total de los trasplantes cardiacos realizados en el mundo, ${ }^{2,4}$ aunque se ha reportado hasta $15 \% .{ }^{14}$ Su principal indicación es la vasculopatía del injerto, en 37 a $90.3 \%$. Las otras indicaciones son el rechazo agudo y la disfunción primaria del injerto cardiaco. Se ha incrementado su indicación en pacientes entre 0 y 17 años y en el grupo etario de 18 a 49 años. ${ }^{15}$

En cuanto a la supervivencia después de un retrasplante, el tiempo entre el primer y el segundo trasplante es un factor de riesgo: la supervivencia es de $65 \%$ a un año en quienes el intervalo es menor de dos años, comparada con $75 \%$ en quienes el retrasplante se realizó en un intervalo mayor. ${ }^{4}$ Tsao et al. ${ }^{16}$ demostraron que la mortalidad se incrementa con la inmunosupresión previa prolongada e intensa (que aumenta el riesgo de los pacientes a desarrollar neoplasias maligna), falla previa de otros órganos antes del retrasplante, mayor edad, sexo femenino y cardiopatía isquémica como causa del primer trasplante. Otros factores citados son la cirugía previa, elevados niveles de PRA y efectos colaterales de los inmunosupresores de uso prolongado. ${ }^{15}$ Sin embargo, a largo plazo si se cuida la selección del receptor, Grun et al. insisten en considerar el periodo transcurrido entre el primer trasplante y el retrasplante como un criterio de selección. ${ }^{17}$

Al comparar pacientes con vasculopatía del injerto tratados médicamente con pacientes retrasplantados por la misma causa, se observó mejor resultado en el retrasplante en los pacientes que presentaban disfunción sistólica, sin embargo, se insiste en ser muy cuidadosos en la selección. ${ }^{18}$ En nuestra experiencia, la cirugía previa no ha demostrado ser un factor relevante en el primer trasplante. ${ }^{19}$
El caso descrito es el primero de retrasplante de corazón en México en forma electiva, cuyo resultado fue exitoso. Podemos concluir que este procedimiento es una opción apropiada para casos bien seleccionados, después de analizar cuidadosamente lo factores de riesgo y las condiciones clínicas.

\section{Bibliografia}

1. Careaga-Reyna G, Zetina-Tun H, Lezama-Urtecho CA. Programa de trasplante cardiaco de la Unidad Médica de Alta Especialidad, Hospital General Dr. Gaudencio González Garza del Centro Médico Nacional La Raza. Rev Invest Clin. 2011;63(Supl 1):85-90.

2. Topkara VK, Dang NC, Cheema JR, Barbato R, Cavallo M, Liu JF, et al. A decade experience of cardiac retransplantation in adult recipients. $J$ Heart Lung Transplant. 2005;24(11):1745-1750. DOI: 10.1016/j. healun.2005.02.015

3. Mehra MR, Crespo-Leiro MG, Dipchand A, Ensminger SM, Hiemann NE, Kobashigawa JA, et al. International Society for Heart and Lung Transplantation working formulation of a standardized nomenclature for cardiac allograft vasculopathy-2010. J Heart Lung Transplant. 2010;29(7):717-727. DOI: 10.1016/j.healun.2010.05.017

4. Magee JC, Barr ML, Basadonna GP, Johnson MR, Mahadevan S, McBride MA, et al. Repeat organ transplantation in the United States, 1996-2005. Am J Transplant. 2007;7(5 Pt 2):1424-1433. DOI: 10.1111/j.1600-6143.2007.01786.x

5. Radovancevic B, McGiffin DC, Kobashigawa JA, Cintron GB, Mullen GM, Pitts DE, et al. Retransplantation in 7,290 primary transplant patients: a 10-year multiinstitutional study. J Heart Lung Transplant. 2003;22:862868. DOI: 10.1016/S1053-2498(02)00803-3

6. Pozzi C, d'Alessandro C, Fernandez F, Nguyen A, Pavie A, Leprince P, et al. Who gets a second heart? A current picture of cardiac retransplantation. Transplant Proc 2014; 46; 202-207. DOI: 10.1016/j.transproceed.2013.08.109

7. Srivastava R, Keck BM, Bennett LE, Hosenpud JD. The results of cardiac retransplantation: an analysis of the Joint International Society for Heart and Lung Transplantation/United Network for Organ Sharing Thoracic Registry. Transplantation. 2000;70(4):606-612.

8. Johnson MR, Aaronson KD, Canter CE, Kirklin JK, Mancini DM, Mehra MR, et al. Heart retransplantation. Am J Transplant. 2007;7(9):2075-2081. DOI: $10.1111 / j .1600-6143.2007 .01902 . x$

9. Saito A, Novick RJ, Kiaii B, McKenzie FN, Quantz M, Pflugfelder P, et al. Early and late outcomes after cardiac retransplantation. Can J Surg. 2013;56(1):21-26. DOI: 10.1503/cjs.012511

10. Doshi AA, Rogers J, Kern MJ, Hauptman PJ. Effectiveness of percutaneous coronary intervention in cardiac allograft vasculopathy. Am J Cardiol. 2004;93(1):90-92. DOI: 10.1016/j.amjcard.2003.08.075

11. Taylor DO, Edwards LB, Mohacsi PJ, Boucek MM, Trulock EP, Keck BM, et al. The Registry of the International Society for Heart and Lung Transplantation: twentieth official adult heart transplant report-2003. J Heart Lung Transplant. 2003;22(6):616-624.

12. Patel VS, Radovancevic B, Springer W, Frazier OH, Massin E, Benrey J, et al. Revascularization procedures in patients with transplant coronary artery disease. Eur J Cardiothorac Surg. 1997;11(5): 895-901.

13. Tham EB, Yeung AC, Cheng CW, Bernstein D, Chin C, Feinstein JA. Experience of percutaneous coronary intervention in the management of pediatric cardiac allograft vasculopathy. J Heart Lung Transplant. 2005;24(6):769-773. DOI: 10.1016/j.healun.2004.04.009

14. Copeland JG, Griepp RB, Bieber CP, Billingham M, Schroeder JS, Hunt $\mathrm{S}$, et al. Successful retransplantation of the human heart. J Thorac Cardiovasc Surg. 1977;73(2):242-247.

15. Tjang YS, Tenderich G, Hornik L, Körfer R. Cardiac retransplantation in adults: an evidence-based systematic review. Thorac Cardiovasc Surg. 2008;56(6):323-327. DOI: 10.1055/s-2008-1038662

16. Tsao L, Uriel N, Leitz K, Naka Y, Mancini D. Higher rate of comorbidities after cardiac retransplantation contributes to decreased survival. J Heart Lung Transplant. 2009;28(10):1072-1074. DOI: 10.1016/j.healun.2009.06.004

17. Grun S, Ceruti B, Chiesa P, Díaz P, Manfredi A, Leone R. Retrasplante cardíaco: a propósito del primer caso en Uruguay. Rev Urug Cardiol. 2006;21(2):124-130. Disponible en: http://www.scielo.edu.uy/scielo. php?script=sci_arttext\&pid=S1688-04202006000200004

18. Goldraich LA, Stehlik J, Kucheryavayya AY, Edwards LB, Ross HJ. Retransplant and medical therapy for cardiac allograft vasculopathy: International Society for Heart and Lung Transplantation Registry Analysis. Am J Transplant. 2016;16:301-309. DOI: 10.1111/ ajt. 13418

19. Careaga-Reyna G, Zetina-Tun HJ, Lezama-Urtecho CA, Arellano-Juárez L, Alvarez-Sánchez LM. Trasplante de corazón en pacientes con cirugía cardiaca previa. Cir Cardiov. 2017;24(1):22-25. DOI: 10.1016/j. circv.2016.09.010 